\title{
Nanoparticle adhesion and removal studied by pulsed laser irradiation
}

\author{
Paul Leiderer ${ }^{\mathrm{a}}$, Michael Olapinski ${ }^{\mathrm{b}}$, Mario Mosbacher ${ }^{\mathrm{a}}$, and Johannes Boneberg ${ }^{\mathrm{a}}$ \\ ${ }^{\mathrm{a} D e p a r t m e n t ~ o f ~ P h y s i c s ~ a n d ~ C e n t e r ~ f o r ~ A p p l i e d ~ P h o t o n i c s, ~ U n i v e r s i t a ̈ t ~ K o n s t a n z, ~ U n i v e r s i t a ̈ t s s t r . ~ 10, ~}$ \\ 78464 Konstanz, Germany \\ ${ }^{\mathrm{b}}$ Department of Physics and Center for Nanoscience, Ludwig-Maximilians-Universität München, \\ Geschwister-Scholl-Platz 1, 80539 Munich, Germany
}

\begin{abstract}
The contactless removal of small particles from surfaces by irradiation with intense laser pulses - dubbed laser cleaning - has been used and studied for nearly two decades. Nevertheless, its applicability and the mechanisms involved are still under debate. Here we give first a brief overview on relevant processes, and then present measurements of the velocities of colloidal model particles after detachment under vacuum conditions. We also demonstrate a new Laser Cleaning approach, by which submicrometer particles are removed by laser irradiation of the rear side of the wafers. The particles are detached by an acoustic shock wave traveling to the wafer front side after laser ablation of the rear side. Not only is this promising approach capable of defect free surface cleaning, detailed studies of particle velocities versus laser fluence also allow insight into the different cleaning mechanisms involved. Furthermore, this technique could be applied to determine adhesion energies of particles in the future.
\end{abstract}

Keywords: laser cleaning, particle adhesion

\section{INTRODUCTION}

Adhesion of nanoparticles to surfaces is a phenomenon of fundamental interest in nanoscience, but also bears wide importance for applications in micro- and nanotechnology. In microelectronics, e.g., unwanted nanoparticles with a size comparable to the IC structures may lead to malfunction of the electronic device. An important aspect is here that conventional cleaning methods, e.g. ultrasonic cleaning, become less and less efficient the smaller the particles are, because the relative contribution of adhesion forces - compared to inertial forces - increases. A concept to solve this problem is the so-called laser cleaning process, where already different variants have been developed [1,2]: The basic idea of (dry) laser cleaning (DLC) is simple: due to the thermal expansion of the substrate (e.g. a silicon wafer) upon irradiation with a short laser pulse the particles at the substrate surface will be accelerated, and then because of their inertia detach from the surface when the expansion of the substrate slows down. The adhesion forces to be overcome are mainly caused by van der Waals interaction, and the required acceleration (or rather deceleration when the thermal expansion stops) can be estimated to be in the range of $10^{7} \mathrm{~m} / \mathrm{s}^{2}$ for particle sizes in the range of several hundred nanometers. More detailed considerations indicate, however, that in addition to the homogeneous expansion of the heated surface further effects have to be taken into account. One is based on the optical field enhancement under a particle leading to local ablation of the substrate. Thereupon, the ablated material can expel the particle from the surface. Furthermore, adsorbed water - especially close to the region where the particle is in contact with the substrate - may be evaporated by the laser pulse and thus give rise to particle detachment. The latter effect can be enhanced in the process of "steam laser cleaning" (SLC), where deliberately a thin layer of liquid is condensed on the substrate, which is explosively evaporated by the laser pulse and thus carries the particles away.

In the first part of this article we give a short overview in which relevant contributions to the laser cleaning process are briefly illustrated. For more detailed information and related literature we refer to Ref.[2]. In an attempt to distinguish between the different mechanisms of particle detachment, we present in the second part first results of time of flight measurements of the detached particles, and in particular describe experiments in which the substrate is irradiated by the laser pulse from the back side, giving rise to an acoustic shock wave which can lead to particle removal without the influence of optical near-field effects or water evaporation.

Konstanzer Online-Publikations-System (KOPS)

URL: http://www.ub.uni-konstanz.de/kops/volltexte/2007/2537/

URN: http://nbn-resolving.de/urn:nbn:de:bsz:352-opus-25371 


\section{SHORT OVERVIEW OVER LASER CLEANING RESULTS}

The experiments to be described here were mostly carried out with silicon wavers as substrates and a frequency-doubled Nd:YAG laser $(\lambda=532 \mathrm{~nm}, \mathrm{FWHM} \sim 8 \mathrm{~ns})$ as a light source. The particles were well-defined colloidal spheres with a narrow size distribution, consisting of various materials like polystyrene (PS) or $\mathrm{SiO}_{2}$, and with diameters ranging from 60 to $1000 \mathrm{~nm}$. They were deposited by means of spin coating the colloidal suspension onto the substrate, which under proper preparation conditions leads to a fraction of isolated particles $>95 \%$.

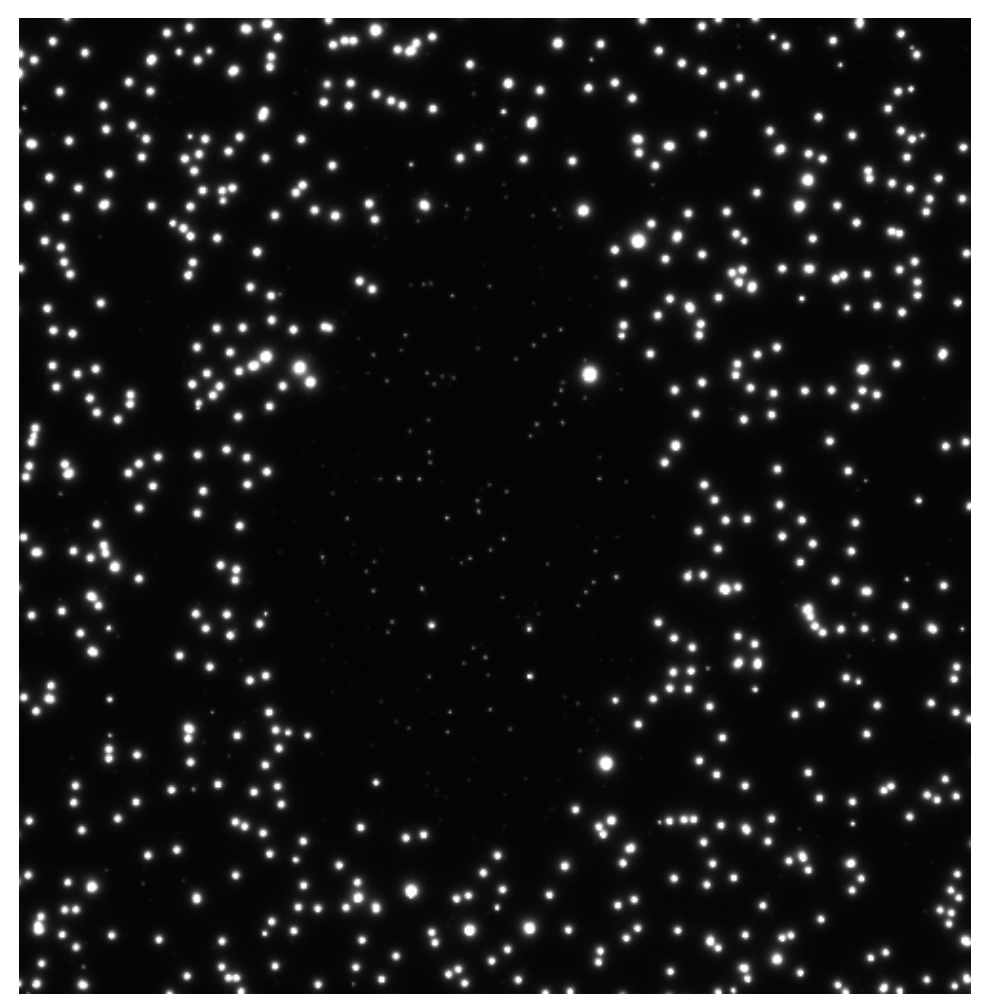

Fig. 1: Optical microscope picture of a Si wafer contaminated with $580 \mathrm{~nm}$ PS particles. In the central black spot the particles have been removed by DLC (Size of the image: $1 \times 1 \mathrm{~mm}^{2}$ ).

A dark field photograph of a Si wafer surface with 580nm PS particles is shown in Fig.1 (the white spots represent the scattered light, not the actual particle size). In the center, where apparently particles are missing, the wafer has been irradiated by a laser pulse with a fluence of $100 \mathrm{~mJ} / \mathrm{cm}^{2}$ in the DLC process. Independent measurements of the surface expansion of laser-exposed Si surfaces by Dobler et al. [3] indicate that the forces due to inertia should in this case be sufficient to overcome adhesion, hence the simple concept of DLC, based on thermal expansion, at first sight appears to work. The laser fluence threshold above which particles are removed is plotted for different particle sizes in Fig. 2. Note that the fact that small particles are apparently more difficult to remove than large ones is also in line with this idea. 


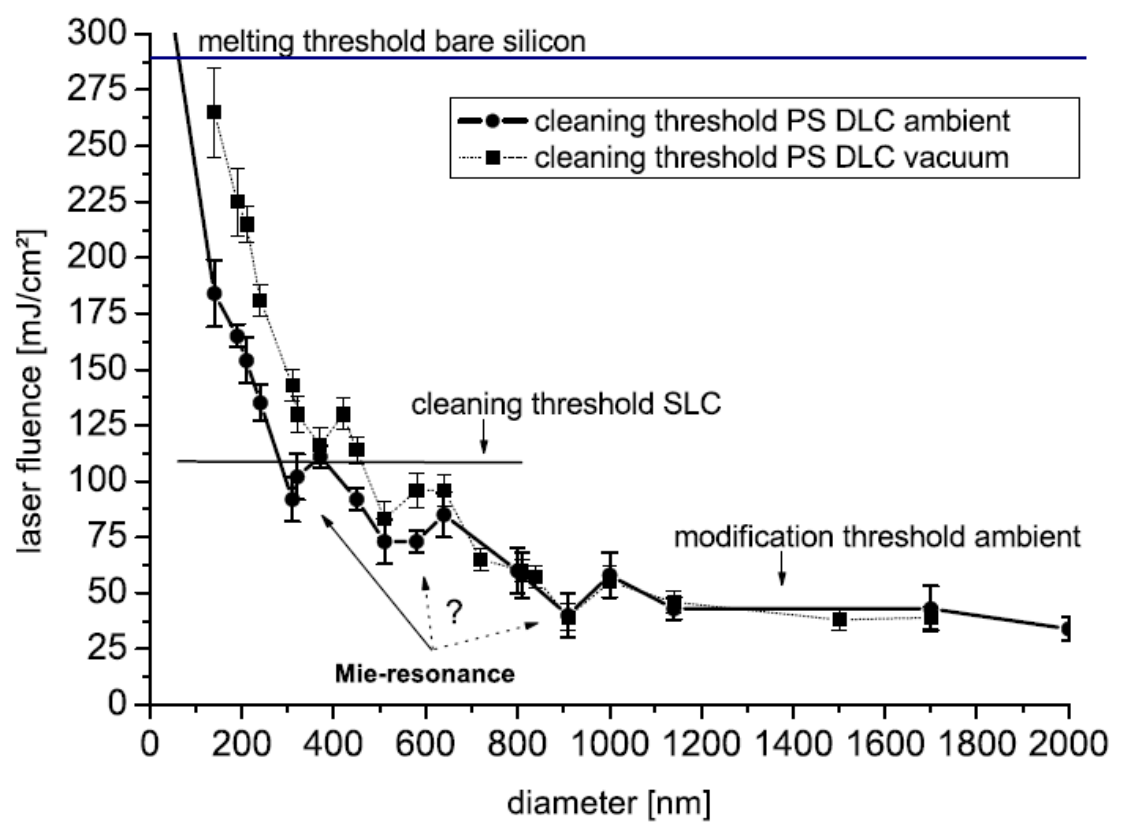

Fig. 2.: DLC thresholds in the applied laser fluence for particle removal in ambient air (circles) and vacuum (squares). Particles smaller than $110 \mathrm{~nm}$ could not be removed. For small particle diameters the threshold in vacuum is distinctly increased compared to air [Ref.4].

A closer inspection of cleaning spots such as the one in Fig.1 shows, however, that the Si surface in this area is not completely flat, but that scattering centers for light are still present (barely visible in the reproduction). More detailed investigations with a scanning electron microscope have revealed ablation holes in the substrate at the original particle positions, caused by the aforementioned local field enhancement underneath the particles. In the case of nanosecond laser pulses this hole structure is rather washed out, and for shorter pulses, where thermal conduction plays a negligible role, it becomes sharper (see Fig.3) [5]. 


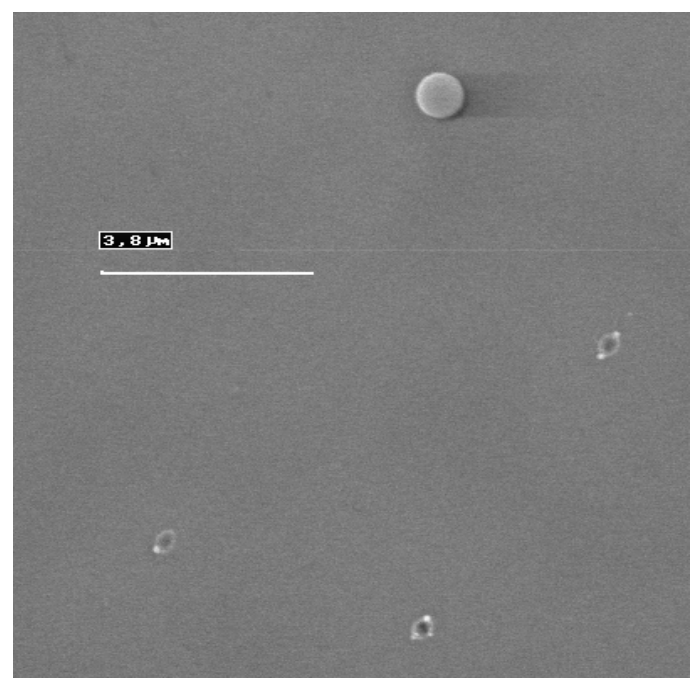

Fig. 3: Scanning electron micrograph of an area cleaned by a picosecond laser pulse (DLC, FWHM $=30 \mathrm{ps}, \lambda=583 \mathrm{~nm}$ ) in a sample with $800 \mathrm{~nm}$ PS spheres. Elliptical holes can be found all over the illuminated region; a remaining particle can also be seen [Ref. 5].

Although these data clearly demonstrate that under certain conditions local ablation in the enhanced near-field of a particle plays a prominent role, there is experimental evidence for other contributions as well. One is the influence of adsorbed water, illustrated by comparing data taken at ambient conditions and in vacuum. Such a comparison is given in Fig. 2 shown already before. Obviously in vacuum the threshold is increased, an effect which is most pronounced for the smaller particles $<400 \mathrm{~nm}$. This demonstrates that under ambient conditions "dry laser cleaning" can considerably be affected by adsorbed water.

In the case of "steam laser cleaning" this effect is enhanced by condensing a thin layer of liquid onto the substrate to be cleaned, as already mentioned in the introduction. The liquid to be chosen should wet the substrate (as does a waterisopropanol mixture on silicon) in order to avoid the formation of droplets, which again could give rise to optical near field damages. Typical film thicknesses are in the range of some hundred nanometers. Under these conditions, universal cleaning thresholds are found, irrespective of the size and the material of the particles to be removed. An example is given in Fig. 4.

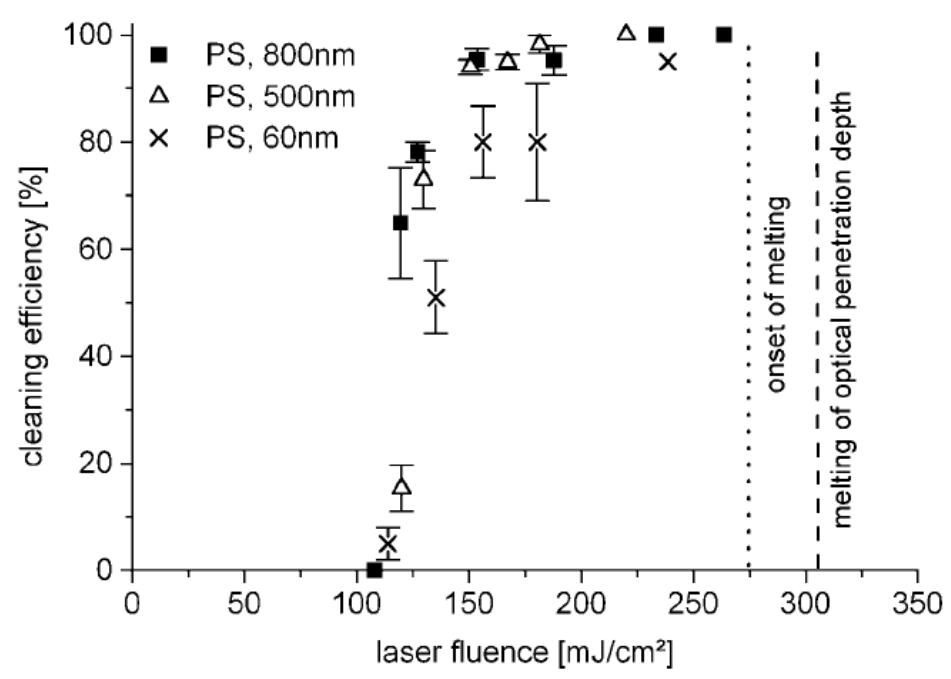

Fig. 4: Experimentally determined cleaning efficiency as a function of applied laser fluence for various PS spheres. The cleaning threshold is size-independent and exhibits a steep increase above the onset of cleaning [Ref. 6] 


\section{BALLISTIC MEASUREMENTS OF PARTICLE DETACHMENT IN LASER CLEANING}

As shown in the last chapter, there are at least 3 mechanisms which may contribute to the particle detachment in laser cleaning: the thermal expansion of the substrate, local ablation of the substrate, and explosive evaporation of water residues under the particle. Separating the roles of thermal expansion and local ablation is a complex task when the sample is irradiated from the particle-coated (front) side because the particles are always exposed to the incident light field. We have therefore set up an experiment where a rapid elongation of the substrate surface can be achieved without parasitic optical near-field effects: The idea is to use a laser pulse incident on the sample from the opposite (rear) side of the sample to generate an acoustic pulse which propagates through the wafer and - provided the intensity is high enough - will give rise to sufficiently high accelerations and inertial forces that the particles are detached. In order to keep the effect of adsorbed water to a minimum the experiments have been carried out in vacuum.

A sketch of the set-up is shown in Fig. 5. For the sake of comparison with DLC the samples could be irradiated either from the rear or from the front side. As in the previous experiments, the light source was a pulsed frequency-doubled $\mathrm{Nd}$ :YAG laser $(532 \mathrm{~nm}, \mathrm{FWHM}=8 \mathrm{~ns})$. In order to characterize not only cleaning thresholds, but also the surplus (kinetic) energy of the particles leaving the surface, we determined the particle velocities as a function of the incident laser fluence, using a time of flight method, where the time delay between the laser pulse and the arrival of ejected particles at a certain distance from the surface is measured [7]. For this purpose, a thin "sheet of light" (SoL) is generated in front of and parallel to the sample surface. When particles are passing through this sheet, light is scattered by them and detected by a photomultiplier. The SoL and photomultiplier detection volume are carefully aligned with respect to the cleaning area and their optics are mounted on translation stages, so that the distance $\delta$ from the sample surface to the SoL can be varied. The sample chamber as a whole, containing the sample, can be moved independently in two axes parallel to the sample surface, so that different spots on the sample can be irradiated and probed.

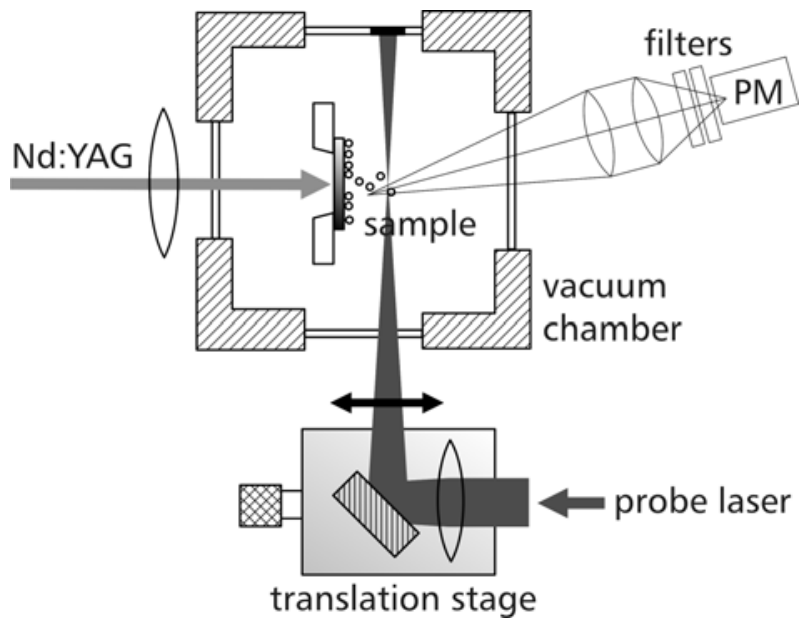

Fig. 5.: Schematic top view of the experimental setup. The sample is placed inside a vacuum chamber and irradiated with a frequency-doubled Nd:YAG laser pulse at the wafer front or rear side. Particles at the wafer front side are detached and leave the surface ballistically. At a certain distance from the sample surface, light from a probe diode laser $(635 \mathrm{~nm}, 25 \mathrm{~mW})$ is focussed by a cylindrical lens to form a thin "sheet of light". When a particle crosses the light sheet, light is scattered and collected by a telescope attached to a photomultiplier. Bandpass filters of the probe laser wavelength protect the photomultiplier from stray light from the Nd:YAG laser. A translation stage is used to vary the position of the light sheet, while the position of the detection telescope is changed in the same way by another translation stage (not shown). The vacuum chamber is also mounted onto a two-axis stage to change the irradiated sample area. 
The scattered light intensity data $I(t)$ versus time $t$ delivered by the photomultiplier is recorded with a digital storage oscilloscope, averaged over 3 to 7 pulses, each irradiating a fresh spot on the sample, and stored for further data treatment.

The $I(t)$ data is converted into velocity profiles according to the equation

$$
n(v) \propto \frac{1}{t} I\left(\frac{\delta}{v}\right)
$$

where $n(v)$ is the relative frequency or number of particles exhibiting velocity $v$. The relation reflects that the intensity of scattered light is proportional to the number of particles crossing the SoL and that the velocity of the particle corresponds to a time of the occurrence of scattered light $t=\delta / v$. The prefactor $1 / t$ accounts for the fact that slower particles have longer dwell times in the scattering volume. Before each set of experiments, the distance $\delta$ between the sample surface and the light sheet is calibrated by recording $I(t)$ traces as described above while moving the SoL-translation stage by defined distances. The linear relation between $\delta$ and the time $t_{m}$ of the maximum intensity shows that the particles indeed move ballistically and their path is not disturbed by collisions and interaction.

Figs. 6 and 7 give examples of the velocity profiles obtained by back and front side irradiation, respectively. Even a superficial comparison of the two figures shows already that there are pronounced differences between the two data sets, indicating that also the details of the detachment mechanisms are different.

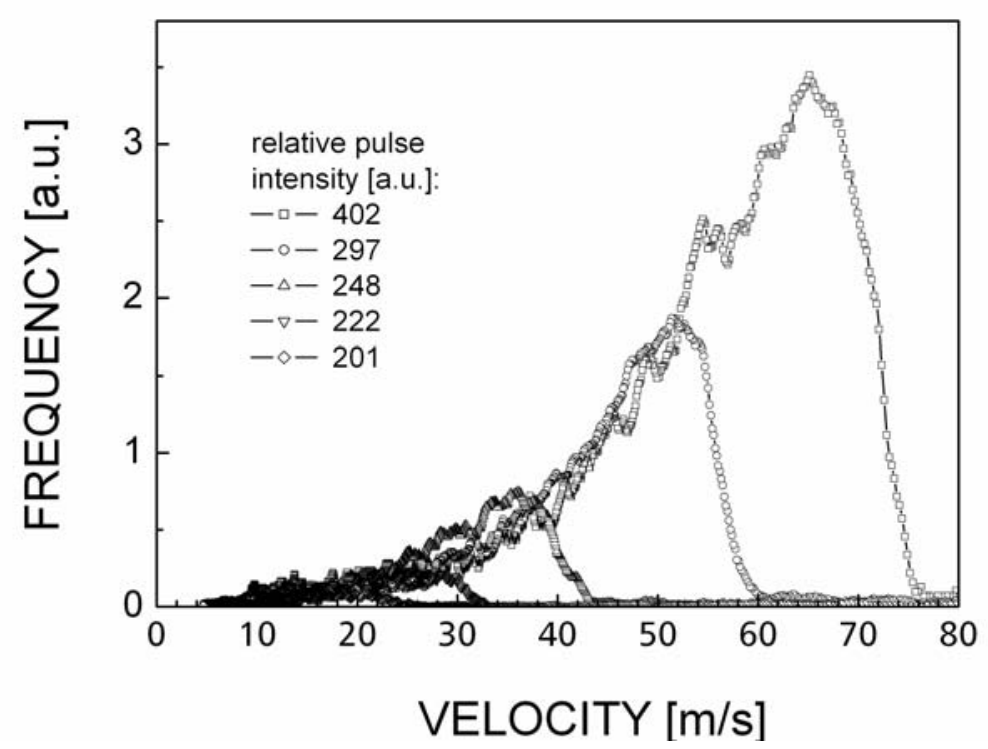

Fig. 6.: Velocity profiles of polystyrene colloids with $840 \mathrm{~nm}$ diameter (PS840) ejected from a silicon wafer at the front side upon back side pulse laser irradiation with various intensities. The frequency axis corresponds to relative particle numbers. For higher pulse intensities the maximum velocity increases as well as the number of particles ejected from the surface (corresponding to the area below the curves). 


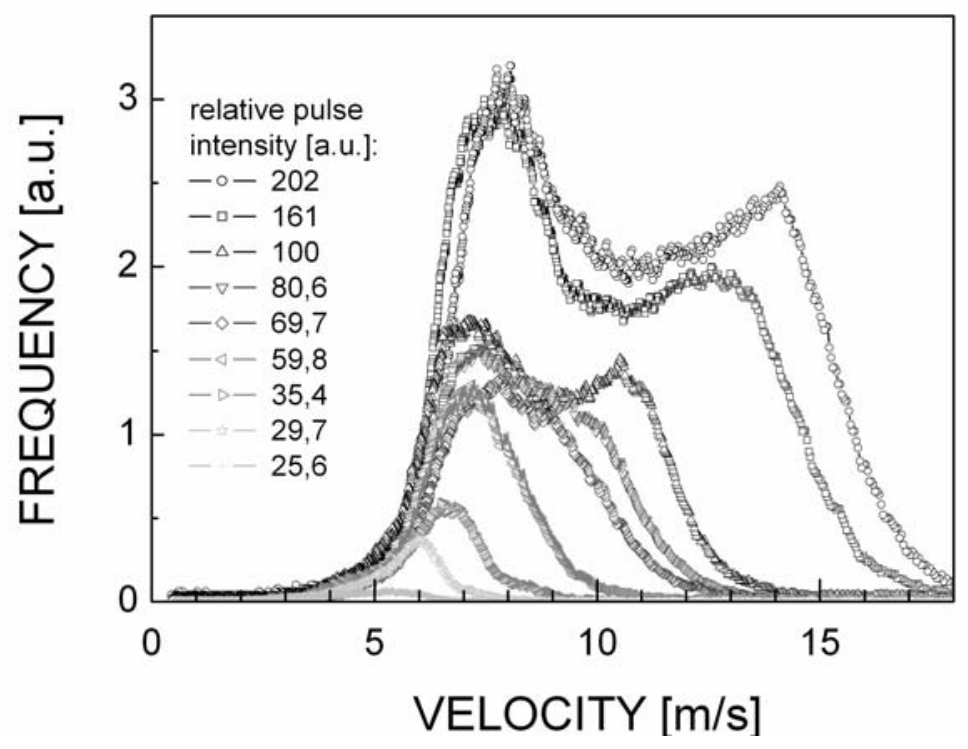

Fig. 7. Velocity profiles for dry laser cleaning (front side irradiation). For low pulse intensities a peak velocity between 5 and $7 \mathrm{~m} / \mathrm{s}$ is reached. For higher intensities a side shoulder develops and grows into a second highvelocity peak, while the first (main) peak essentially remains at a velocity around $7 \mathrm{~m} / \mathrm{s}$.

We first discuss the data for the back side illumination in Fig. 6. The characteristic shape and its evolution with different laser intensities is caused by the Gaussian beam profile of the pulsed laser, resulting in a similar excitation profile on the front side surface. Particles are observed, once the laser intensity exceeds a specific threshold fluence. The maximum velocity of ejected particles is strongly dependent on the pulse energy. For further evaluation, the velocity of the $50 \%$ value of the high-velocity edge of the velocity profile is extracted.

In Fig. 8 these data are plotted against the laser fluence. The lines are square root fits to the data, suggesting a behavior

$$
v \propto\left(F-F_{t h}\right)^{1 / 2}
$$

where $F$ is the incident laser fluence and $F_{t h}$ the threshold at which particle ablation sets in. Hence the kinetic energy of the particles is

$$
E_{k i n} \propto\left(F-F_{t h}\right)
$$

Presently experiments are underway which relate the laser fluence to the surface elongation and acceleration using an interferometric technique similar to the one described by Dobler et al.[3]. Already the first experiments indicate, that the surface velocities measured are distinctly smaller than the particle ejection velocities [8].

It should be pointed out that these data suggest a particle detachment process with a threshold energy that corresponds to the adhesion energy of the particle. It should therefore be possible to use the method to precisely determine adhesion energies of a variety of deposited particles and particle sizes. An example thereof is displayed in Fig. 8: the cleaning threshold of samples with different storage times before the experiment varies significantly. This effect, which has been studied for the DLC threshold previously [9], is clearly demonstrated with the back side shock laser cleaning technique. 


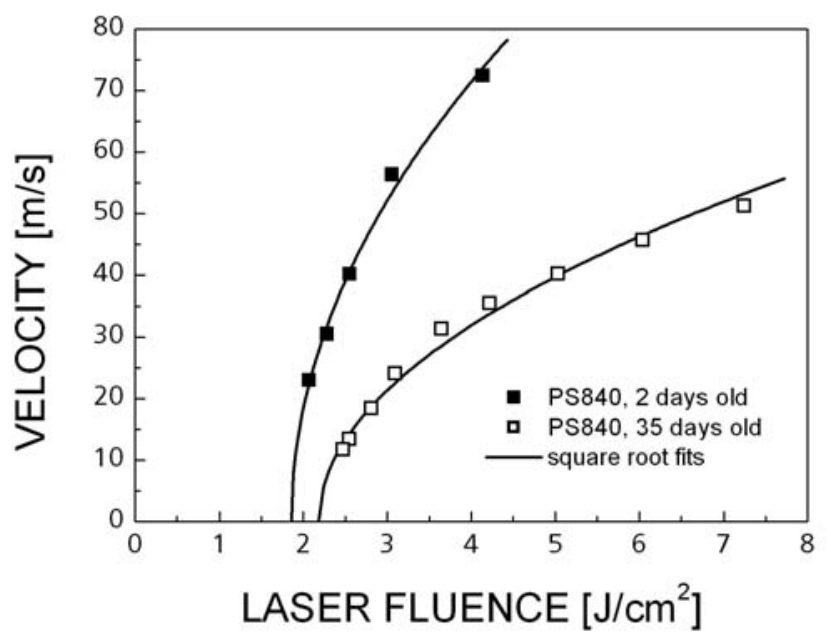

Fig. 8. Velocity at the maximum edge is plotted against the laser fluence for the profiles in Fig. 6 (PS840, full symbols) as well as for similar particles, but on samples that had been stored for a longer time before the experiment (open symbols). Both data points can be very well fit with square root curves. The older sample displays a higher cleaning threshold and lower detachment velocities.

In our lab we were able to successfully remove particles with diameters down to $170 \mathrm{~nm}$ with this method, although this seems not to be the limit. In general, the cleaning limits of this approach are defined by the laser energy thresholds for damage to the front surface. For particularly high fluence, the intensities of the generated shock wave could even damage the wafer interior. As a first evaluation of hypothetical surface damages, samples with particles 580nm in diameter have been investigated by AFM after particle detachment. No damage could be found as opposed to DLC , where small holes remain after the cleaning process due to local ablation and optical near field enhancement.

We now turn to a discussion of Fig. 7, where particle velocities are displayed for front side laser irradiation, the usual DLC. Interestingly, the data for higher fluence exhibit two peaks in the particle frequency versus velocity. When increasing the laser fluence, the position of the first peak remains roughly unaltered, but a second shoulder at higher velocities develops and grows into a second peak. These data indicate already that two different mechanism of detachment are involved, in which the fluence dependent peak shows similarities to the shock wave cleaning behaviour, while the origin of the fluence-indepent part still has to be clarified. This once again shows the complexity of the DLCprocess. We want to point out, however, that also in this case of DLC the detachment velocity is distinctly larger than the expansion velocity of the surface (without particles) as determined by Dobler et al. [3].

\section{SUMMARY}

An experimental setup has been introduced for the measurement of the velocities of particles ejected from a substrate after irradiation of the front or back side with a laser pulse. The measured particle velocities range between 5 and 100 $\mathrm{m} / \mathrm{s}$. It has been shown that the measured data and the comparison between front and back side irradiation allows new insight into the mechanisms involved and raises interesting new questions. A new Laser cleaning approach has been introduced: back side shock laser cleaning. The successful cleaning of particles down to 170nm in diameter could be demonstrated. This approach allows the separation of the laser energy input from the particle detachment process and thus reduces the complexity of the cleaning process. In this way, the combination of new techniques offers an experimental approach to determine adhesion energies of particles on surfaces and a chance to compare these non- 
equilibrium dynamic adhesion energies to the slow equilibrium values measured with techniques based on other principles such as atomic force microscopy [10].

\section{ACKNOWLEDGEMENTS}

The authors would like to thank Florian Lang, Johannes Graf, Tobias Geldhauser, Florian Ziese and Pascal Frank for valuable discussions. We acknowledge financial support by the Deutsche Forschungsgemeinschaft within the SFB 513 and the Center of Advanced Photonics at the University of Konstanz.

\section{REFERENCES}

1. W. Zapka, W. Ziemlich and A. C. Tam, Appl. Phys. Lett. 58, 2217 (1991).

2. B. Luk'yanchuk, editor, "Laser Cleaning” World Scientific, Singapore 2002 and references therein

3. V. Dobler, R. Oltra, J.P. Boquillon, M. Mosbacher, J. Boneberg, P. Leiderer, Appl. Phys. A 69 S335 (1999).

4. M. Mosbacher, H.-J. Münzer, M. Bertsch, O. Dubbers, V. Dobler, T. Mühlberger, G. Schrems, J. Boneberg, D. Bäuerle, B.-U. Runge, P. Leiderer, Proc SPIE 4426, 308 (2002)

5. M. Mosbacher, H.-J. Münzer, J. Zimmermann, J. Solis, J. Boneberg, P. Leiderer, Appl. Phys. A 72, 41 (2001)

6. M. Mosbacher, V. Dobler, J. Boneberg, P. Leiderer, Appl. Phys. A 70, 669 (2000)

7. A. Habenicht, M. Olapinski, F.Burmeister, P.Leiderer, J. Boneberg, Science 309, 2043-2045 (2005)

8. T. Geldhauser, Diploma thesis, University of Konstanz, 2006

9. G. Schrems, M.P. Delamare, N. Arnold, P. Leiderer, D. Bäuerle, Appl. Phys. A 76, 847(2003)

10. L.O. Heim, J. Blum, M. Preuss, H.J. Butt, Phys. Rev. Lett. 83, 3328 (1999) 